\title{
When it helps to be misled: Facilitative effects of increasing the frequency of conflicting stimuli in a Stroop-like task
}

\author{
GORDON D. LOGAN \\ Erindale College, University of Toronto, Mississauga, Ontario L5L 1C6, Canada \\ and \\ N. JANE ZBRODOFF \\ Ontario Institute for Studies in Education, University of Toronto, Ontario M5S IV6, Canada
}

\begin{abstract}
Three experiments are reported that involve responding to the meaning or position of a word (ABOVE or BELOW) presented above or below a fixation point. Position and word meaning conflicted (ABOVE/below or BELOW/above) or were compatible (ABOVE/above or BELOW/below), and the relative frequency of conflicting trials was varied. Experiment 1 required responses to the word and its position. Compatibility and frequency had no effect in the spatial task, but interacted strongly in the word task: Compatible stimuli were processed faster when conflicting trials were rare (20\% conflicting), but conflicting stimuli were processed faster when they were frequent (80\% conflicting). Experiments 2 and 3 used the word task only and extended these findings to intermediate $(20 \%, 40 \%, 60 \%$, and $80 \%$ conflicting) and more extreme $(10 \%, 20 \%, 80 \%$, and $90 \%$ conflicting) frequencies, respectively. The advantage for conflicting stimuli when they were frequent was taken as evidence for a strategy involving dividing attention between reported and unreported dimensions.
\end{abstract}

This paper reports an investigation of strategies for performing a Stroop-like task. It is based on the principle that performance in new task environments depends on a flexible yet predictable allocation of existing cognitive resources: The organization of existing resources can be changed quickly to do what the new task requires, and the strategy chosen will best exploit the regularities of the task environment to optimize performance (cf. Logan, 1978; Posner \& Snyder, 1975a). The experiments were designed to determine whether such flexibility is possible in Stroop-like tasks.

The Stroop task involves reporting one dimension of a multidimensional stimulus. When an unreported dimension signals a meaning that seems relevant to the task but conflicts with the meaning of the reported

The order of authorship was determined randomly. This research was supported by Grants A0682 and E4209 to Gordon Logan from the National Research Council of Canada. Jane Zbrodoff was supported by an Ontario Graduate Scholarship. We thank Gail McKoon and Roger Ratcliff for valuable comments and criticism, and Kathy Constantinou for conducting Experiment 3. We are especially grateful to Doug Mewhort for providing his display program and making it run on our computer, and for hints and encouragement along the way. Requests for reprints may be sent to Gordon D. Logan, Department of Psychology, Erindale College, University of Toronto, Mississauga, Ontario L5L 1C6, Canada, or to N. Jane Zbrodoff, Department of Applied Psychology, Ontario Institute for Studies in Education, 252 Bloor Street West, Toronto, Ontario M5S 1 V6, Canada. dimension, interference results. For example, colornaming time is increased substantially when the colored forms are words that specify incompatible colors (Keele, 1972; Stroop, 1935), and reading time for words that specify spatial positions is increased when the words are presented in positions that conflict with their meanings (Palef, 1978; Palef \& Olson, 1975). In general, if two dimensions can specify the same meaning, the one that is processed faster will interfere with the one that is processed slower, but not vice versa (Palef \& Olson, 1975; for a general review, see Dyer, 1973).

The Stroop task was chosen for the present investigation because the literature is nearly unanimous in assuming that it is performed in only one way, by attending selectively to the reported dimension. Interference from conflicting meanings on unreported dimensions is typically interpreted as reflecting some fixed, strategy-invariant aspect of human information processing; conflicting meanings become available to influence the decision because selective attention fails (Treisman, 1969) or because unreported dimensions are processed automatically (Keele, 1972; Posner \& Snyder, 1975a). In contrast, we believe that selective attention to the reported dimension is only one strategy for performing the task, and we demonstrate in the experiments reported here that different strategies can produce the same effects. In view of the widespread belief that Stroop interference is strategy invariant, this demonstration is strong evidence that the organiza- 
tion of cognitive resources can be flexible and responsive to significant aspects of the task environment.

The experiments involved words that specified spatial positions (ABOVE and BELOW) presented in compatible and conflicting positions (above or below the fixation point). Either the word or the spatial position was to be reported as quickly as possible. The "correlation" between reported and unreported dimensions was varied by manipulating the relative frequency of trials on which the two dimensions specified conflicting meanings. Different predictions about the effects of this manipulation on speed and accuracy can be derived from three strategies that subjects might adopt to perform the task. The first strategy involves selective attention to the reported dimension and corresponds to the standard view of Stroop performance. The second strategy involves dividing attention between reported and unreported dimensions, and the third involves selective attention to the unreported dimension.

Predictions about the effects of varying the frequency of conflicting trials can be developed and compared by expressing the three strategies in terms of a random-walk model of speeded decision making (Pachella, 1974). In random-walk models, evidence relevant to the decision is accumulated over time and compared to thresholds representing each alternative. In two-choice situations (like the present task), evidence for one alternative is evidence against the other, so the current state of evidence can be represented as a point on a decision axis between thresholds representing the two alternatives. As information accumulates over time, the current state of evidence is driven along the decision axis toward one threshold and away from the other; once it crosses a threshold, the appropriate response is emitted. Reaction time (RT) depends on the duration of the random walk, which is determined by the amount of evidence required to cross a threshold (i.e., the position of the thresholds relative to the initial state of evidence) and the rate at which evidence can be accumulated.

The Stroop situation can be modeled by allowing evidence from the unreported dimension to influence the decision about the reported one. When the unreported dimension can be processed much faster than the reported one (as in the present studies), information about the unreported dimension may be viewed as shifting the initial state of evidence about the reported dimension toward one threshold or the other. ${ }^{1}$ Conflicting stimuli would shift it away from the correct threshold, so that more evidence (and thus more time) would be necessary to respond correctly. Compatible stimuli would shift the initial state of evidence toward the correct threshold and thus save time.

This model requires that the current state of evidence bearing on the decision be expressed as a weighted sum of the evidence available about the reported dimension and the evidence available about the unreported dimension (i.e., the greater the weight, the stronger the contribution to the current state of evidence). The idea of attaching weights to evidence is very important. It allows us to differentiate attentive and automatic processing in terms of properties of the weights and to express the three strategies as different combinations of weights.

A major difference between attending and processing automatically is that attention is under voluntary control, while automatic processing is not (Posner \& Snyder, 1975a). Thus, weights that represent attending may vary in magnitude and sign according to the current strategy to allow a flexible blending of information (changes in sign allow responses opposite to habitual meanings, e.g., responding "below" to a word presented above the fixation point). ${ }^{2}$ By contrast, weights that represent automatic processing would be fixed in magnitude and sign, depending on the degree of practice and the strength and direction of the correlation between the habitual meaning and the meaning relevant to the task.

In this framework, selective attention to the reported dimension amounts to computing a weight and assigning it to evidence available about the reported dimension through an act of attention. The weight attached to the unreported dimension would be assigned automatically, subject to the above constraints, not as an act of attention. This strategy corresponds to the view of Stroop performance most commonly held in the literature (e.g., Kahneman, 1973; Posner \& Snyder, 1975a; Treisman, 1969). Since subjects have no control over the weights assigned automatically to the unreported dimension, this strategy must produce interference whenever conflicting stimuli are presented

It may be possible to diminish the influence of the unreported dimension by increasing the weight assigned to the reported dimension, but the influence can never be removed entirely. At best, it may become smaller than measurement error. Note that the weight attached to the reported dimension or the thresholds (or both) must be adjusted to prevent responses based on evidence available automatically from the unreported dimension.

Given selective attention to the reported dimension, increasing the frequency of conflicting trials may have no effect on the interference observed, or the interference may be mitigated somewhat if the weight on the reported dimension is increased proportionately. With this strategy, however, it is not possible to process conflicting stimuli faster than compatible stimuli. The second strategy differs in this regard.

Dividing attention between dimensions amounts to computing and assigning weights to each dimension through an act of attention. The weights would have the same sign (positive) when compatible stimuli were more frequent, and opposite signs (positive for reported, negative for unreported) when conflicting stimuli were more frequent. Thus, RT would be faster with 
conflicting stimuli than with compatible ones whenever conflicting stimuli were more frequent. The unreported dimension might be processed automatically as well and receive additional weight in the decision process. In order to benefit from overcoming habitual response tendencies, the weight assigned attentionally to the unreported dimension must be larger (absolutely) than the weight assigned automatically, but it must remain small enough that it does not produce a response without some information from the reported dimension. Otherwise, the strategy would produce errors whenever stimuli were inconsistent with expectation. The third strategy differs in this regard.

Selective attention to the unreported dimension amounts to assigning it a weight in the decision process through an act of attention. The weight would be positive if compatible stimuli were more frequent and negative if conflicting stimuli were more frequent. Weight could be assigned automatically to the "reported" dimension, but this would have little effect since, presumably, the unreported dimension could be processed faster. This strategy would allow RTs as fast as those to the unreported dimension. The responses it produced would be accurate whenever stimuli matched expectation (i.e., positive weights for compatible stimuli, negative weights for conflicting stimuli) and inaccurate whenever stimuli were inconsistent with expectation (i.e., positive weights for conflicting stimuli, negative weights for compatible stimuli). Given the usual requirement to minimize errors, this strategy is not likely to be adopted unless nearly all stimuli are of one type (compatible or conflicting). Nevertheless, there is some evidence that subjects will use this strategy when it can produce an acceptable level of accuracy (see Dixon, 1978).

The first experiment reported here required subjects to respond to the word and to spatial position. The relative frequency of conflicting trials was varied in both tasks. On the basis of previous work (Palef, 1978; Palef \& Olson, 1975), responses to spatial position were expected to be faster than responses to the word, so interference was expected only in the word task. The pattern of interference across frequency conditions should reveal the strategy used to perform the task: Selective attention to the reported dimension would be indicated if RTs were slower with conflicting stimuli than with compatible stimuli for all frequencies of conflicting trials. Divided attention between dimensions would be indicated if RTs were slower with conflicting stimuli than with compatible stimuli when conflicting trials were relatively rare, but faster with conflicting stimuli than with compatible stimuli when conflicting trials were relatively frequent (i.e., a "crossover" interaction between frequency and type of trial). Selective attention to the unreported dimension would be indicated if RTs to words were nearly as fast as those to spatial position and if nearly all responses to the less frequent stimulus type were errors.

\section{EXPERIMENT 1}

\section{Method}

Subjects. Eight volunteers from the summer laboratory staff at Erindale College served as subjects. Six were female and two were male.

Apparatus and Stimuli. The stimuli were the words ABOVE and BELOW written in capital letters and presented above and below a central fixation point. The words were displayed on a point-plot cathode-ray tube (Tektronix Model 604 equipped with $P_{31}$ phosphor) under the control of a PDP-11/03 laboratory computer (Digital Equipment Corporation). Each letter was formed by illuminating points in a 5 by 7 dot matrix and subtended about $.43 \times .57 \mathrm{deg}$ of visual angle when viewed at a distance of $60 \mathrm{~cm}$. Each word subtended about $2.68 \mathrm{deg}$ horizontally and $.57 \mathrm{deg}$ vertically and appeared $2.77 \mathrm{deg}$ above or below the center of the screen.

Each trial began with a fixation point illuminated in the center of the screen for $500 \mathrm{msec}$. It was then extinguished and replaced with the word for that trial. The word remained on the screen until the subject responded. The response initiated an intertrial interval of at least $2.5 \mathrm{sec}$. The duration varied somewhat due to variable requirements to access the computer's disks between trials. The computer measured RT in milliseconds from the onset of the word to the onset of the response and recorded the response for each trial. Subjects responded by pressing the leftmost or the rightmost of a panel of eight telegraph keys with the index fingers of their left and right hands, respectively. They rested their fingers on the keys between trials.

Procedure. Each subject performed two tasks. In the spatial task, the subjects pressed buttons to indicate whether the word was presented above or below the fixation point. In the word task, they pressed to indicate whether the word itself was ABOVE or BELOW. In both tasks, half of the subjects pressed the right button to indicate "above" and the left button to indicate "below," while the other half did the opposite.

Each task was performed for two consecutive blocks, one in which $20 \%$ of the trials were conflicting and one in which $80 \%$ of the trials were conflicting. The order of tasks and the order of frequency conditions within tasks were balanced orthogonally between subjects. Each subject received the frequency conditions in one task in the same order as in the other.

A block consisted of 100 trials, 20 trials with one stimulus type (compatible or conflicting) and 80 with the other. Within stimulus types, each of the two tokens appeared equally of ten (i.e., ABOVE/above was as frequent as BELOW/below, and $\mathrm{ABOVE} / \mathrm{below}$ was as frequent as BELOW/above). Within these constraints, the order of stimuli was random. A separate random order was constructed for each block for each subject.

Instructions stressed both speed and accuracy. Subjects were shown examples of each stimulus type and token and told how to respond in each task. Once it was clear they understood the instructions, testing began. No practice was given. Subjects were allowed short breaks between blocks if they wished.

\section{Results}

Spatial task. The mean RTs for correct responses in each combination of trial type (compatible or conflicting) and frequency (20\% or $80 \%$ conflicting) are plotted in Figure 1.

From the figure, it is clear that frequency had no effect on RT $[F(1,7)<1]$. Trial type had a weak effect, compatible RTs were $14 \mathrm{msec}$ faster than conflicting ones, but the effect was not significant statistically $[F(1,7)=3.13, p>.10]$. The interaction between 


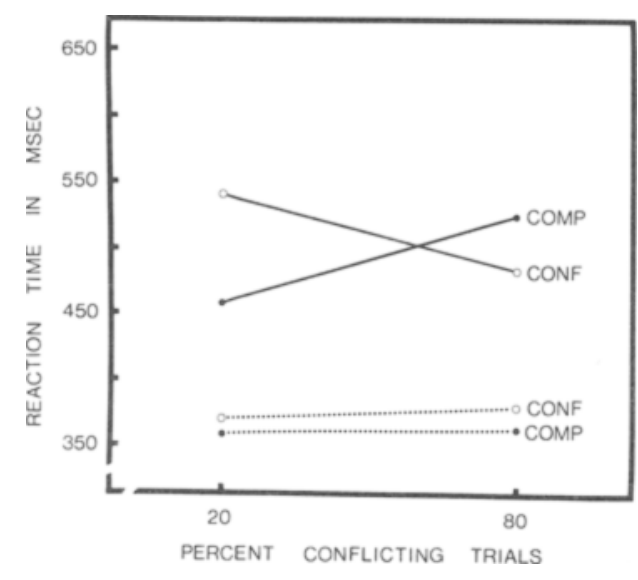

Figure 1. Reaction time as a function of the relative frequency of conflicting trials (percentage) for the spatial task (broken lines) and the word task (solid lines) in Experiment 1. (Trial type is the parameter: Filled circles = trials with compatible stimuli; open circles $=$ trials with conflicting stimuli.)

trial type and frequency was not significant either $[F(1,7)<1]$.

The error data, presented in Table 1 , show similar trends.

Word task. Mean RTs for correct responses in each combination of trial type and frequency are plotted in Figure 1. Clearly, RTs to the words were much slower than those to spatial position (by $113 \mathrm{msec}$ ). In this task, trial type appears to have had a strong effect, modulated by the frequency of conflicting trials. When conflicting trials were relatively rare, RTs to conflicting stimuli were substantially slower (by $81 \mathrm{msec}$ ) than those to compatible stimuli. However, when conflicting trials were relatively frequent, the opposite held: RTs to conflicting stimuli were substantially faster than those to compatible stimuli (by $42 \mathrm{msec}$ ). This crossover was apparent in the individual data of six subjects; the other two subjects showed a reduction in the advantage for compatible stimuli as the frequency of conflicting trials increased. In the group data, the crossover effect was so strong that neither the main effect of trial type $[F(1,7)=2.28, p>.10]$ nor that of frequency
$[F(1,7)<1]$ was significant, although the interaction between them was highly significant $[F(1,7)=43.94$, $\mathrm{p}<.01$.

The error data, presented in Table 1, reflect these trends. Note, however, that error rates were generally low, even with the infrequent stimuli.

\section{Discussion}

There were no effects of trial type or frequency of conflicting trials in the spatial task because information about spatial position was available some $113 \mathrm{msec}$ before information about word meaning (Palef \& Olson, 1975). Instead, the effects appeared in the word task, and their pattern suggests that subjects had divided attention between dimensions to perform the task. The advantage for conflicting stimuli found when they were relatively frequent (i.e., the $80 \%$ condition) is not possible given selective attention to word meaning, but follows as a natural consequence of dividing attention between dimensions. Further, the error rate on infrequent trials was too low (mean $=.066$ ) for focusing attention on the unreported dimension to be a serious possibility, although it follows as a consequence of dividing attention between dimensions. The large difference between spatial and word RTs is also inconsistent with focusing attention on the unreported dimension.

It is interesting that the crossover was not sym. metrical. Facilitation and interference were substantially smaller when conflicting stimuli were relatively frequent ( $80 \%$ condition). This may reflect automatic processing of position information so that it receives additional weight in the decision process. Since the sign of the automatic weight is fixed, it would facilitate compatible trials and interfere with conflicting ones, and so destroy the symmetry of the crossover effect. Alternatively, subjects may be more reluctant to attach large negative weights than large positive weights, so facilitation and interference would be greater when positive weights were appropriate (i.e., in the $20 \%$ condition).

The three strategies have been presented as different, discrete approaches to the task. It seems more likely that they result from continuous variation in a single approach. If a constant attentional weight is attached

Table 1

Error Rates (Proportion) for Each Combination of Trial Type and Frequency of Conflicting Trials (Percent) in Experiments 1, 2, and 3

\begin{tabular}{|c|c|c|c|c|c|c|c|c|}
\hline \multirow{2}{*}{$\begin{array}{c}\text { Experi- } \\
\text { ment }\end{array}$} & \multirow[b]{2}{*}{ Task } & \multirow{2}{*}{$\begin{array}{l}\text { Trial } \\
\text { Type }\end{array}$} & \multicolumn{6}{|c|}{ Frequency } \\
\hline & & & 10 & 20 & 40 & 60 & 80 & 90 \\
\hline 1 & Spatial & $\begin{array}{l}\text { Compatible } \\
\text { Conflicting }\end{array}$ & & $\begin{array}{l}.014 \\
.044\end{array}$ & & & $\begin{array}{l}.006 \\
.020\end{array}$ & \\
\hline 1 & Word & $\begin{array}{l}\text { Compatible } \\
\text { Conflicting }\end{array}$ & & $\begin{array}{l}.001 \\
.081\end{array}$ & & & $\begin{array}{l}.050 \\
.016\end{array}$ & \\
\hline 2 & & $\begin{array}{l}\text { Compatible } \\
\text { Conflicting }\end{array}$ & & $\begin{array}{l}.025 \\
.200\end{array}$ & $\begin{array}{l}.033 \\
.050\end{array}$ & $\begin{array}{l}.050 \\
.058\end{array}$ & $\begin{array}{l}.150 \\
.030\end{array}$ & \\
\hline 3 & & $\begin{array}{l}\text { Compatible } \\
\text { Conflicting }\end{array}$ & $\begin{array}{l}.012 \\
.090 \\
\end{array}$ & $\begin{array}{l}.009 \\
.060\end{array}$ & & & $\begin{array}{l}.060 \\
.015\end{array}$ & $\begin{array}{l}.160 \\
.011\end{array}$ \\
\hline
\end{tabular}


to the reported dimension, the three strategies can be produced by increasing the magnitude of the attentional weight on the unreported dimension from zero (selective attention to the reported dimension), through intermediate values (divided attention between dimensions), to a point at which information from the unreported dimension is sufficient to produce a response (selective attention to the unreported dimension). Performance can be optimized if the weight attached to the unreported dimension is made proportional to its validity as a cue to the response appropriate to the reported dimension. This principle is well established in other contexts (in particular, see Neely, 1977; Posner \& Snyder, 1975b). In the present experiments, the validity of position information as a cue to word meaning depends on the relative frequency of conflicting trials, increasing from zero as relative frequency differs from .5. If the attentional weight on position information is adjusted in proportion to cue validity, the amount of facilitation and interference observed should increase as the relative frequency of conflicting trials differs from .5. The second and third experiments were designed to test this proposition. Both used the word task only, and both involved four blocks, each with a different relative frequency of conflicting trials. Both replicated the $20 \%$ and $80 \%$ conditions of Experiment 1 . In addition, Experiment 2 examined intermediate relative frequencies (i.e., $40 \%$ and $60 \%$ conditions), and Experiment 3 examined more extreme relative frequencies (i.e., $10 \%$ and $90 \%$ conditions).

\section{EXPERIMENT 2}

\begin{abstract}
Method
Subjects. Eight volunteers from the population sampled in Experiment 1 served as subjects. Six were female and two were male. None had served in Experiment 1.

Procedure. The apparatus and stimuli were the same as in Experiment 1. The procedure was the same as Experiment 1 (i.e., four blocks of 100 trials) except for three changes: First, only the word task was used. Second, in different blocks, 20,40, 60 , and 80 of the 100 trials involved conflicting stimuli. The order of frequency conditions varied between subjects according to a 4 by 4 balanced Latin square and was orthogonal to the assignment of stimuli to responses. Third, to minimize transition effects, subjects were told the relative frequency of conflicting trials (expressed to them as a percentage) just before beginning each block.
\end{abstract}

\section{Results}

Mean RTs for correct responses in each combination of trial type and frequency are plotted in Figure 2. Again, there was an effect of trial type modulated by the effect of frequency. RTs to compatible stimuli increased monotonically with the relative frequency of conflicting trials, while RTs to conflicting stimuli decreased monotonically, replicating the crossover observed in Experiment 1. Once again, the crossover was not symmetrical: The difference between compatible and conflicting trials in the $80 \%$ condition $(58 \mathrm{msec})$

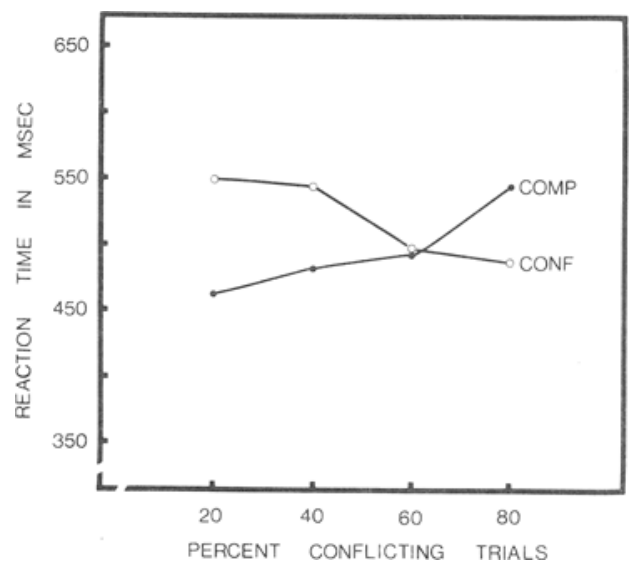

Figure 2. Reaction time as a function of the relative frequency of conflicting trials (percentage) in Experiment 2. (Trial type is the parameter: Filled circles $=$ trials with compatible stimuli; open circles $=$ trials with conflicting stimuli.)

was smaller than the difference in the $20 \%$ condition $(90 \mathrm{msec})$ and about the same as the difference in the $40 \%$ condition $(62 \mathrm{msec})$. The difference in the $60 \%$ condition was negligible ( $5 \mathrm{msec}$ ).

Seven out of eight subjects showed a monotonic decrease in the difference between conflicting and compatible RTs as the relative frequency of conflicting trials increased. The one anomalous subject showed a larger difference in the $40 \%$ condition than in the $20 \%$ condition, but showed a monotonic decrease otherwise. In this experiment, seven out of eight subjects showed an advantage for conflicting stimuli when they appeared on $80 \%$ of the trials.

Analysis of variance supported these conclusions. Frequency had no significant main effect $[F(3,21)=2.18$, $\mathrm{p}>.10 \mathrm{l}$, and trial type had a weak, although significant, main effect $[F(1,7)=6.17, p<.05]$. As in Experiment 1, however, the interaction between frequency and trial type was highly significant $[F(3,21)=55.37, p<.01]$.

The hypothesis that weights are adjusted to optimize performance was tested by a contrast that compared the facilitation and interference in the $20 \%$ and $80 \%$ conditions with that observed in the $40 \%$ and $60 \%$ conditions (i.e., infrequent RT - frequent RT). The contrast was highly significant $[F(1,21)=26.64, p<.01]$, indicating more facilitation and interference in the extreme frequency conditions than in the intermediate ones.

The error data, presented in Table 1 , reflect similar trends. Note, however, the relatively high error rates with infrequent stimuli in the extreme frequency conditions (mean $=.175$ ).

\section{Discussion}

This experiment replicated the crossover interaction observed in Experiment 1 and extended it to intermediate relative frequencies ( $40 \%$ and $60 \%$ conditions), 
suggesting again that subjects performed the task by dividing attention between dimensions. However, the relatively high error rates with infrequent stimuli in the extreme frequency conditions suggest that on some trials subjects may have focused attention exclusively on the unreported dimension. Such selective attention may have occurred on some $15 \%$ of the trials in the extreme frequency conditions (estimated from the difference in error rates with frequent and infrequent stimuli). This does not account for the increased interference observed as relative frequency changed from intermediate to extreme values, and it is likely that on the remaining trials $(85 \%)$, subjects divided attention between dimensions. The disadvantage for compatible stimuli when $80 \%$ of the trials were conflicting cannot be explained as selective attention to either dimension.

The greater facilitation and interference observed with extreme frequencies suggests that subjects optimized performance by adjusting the weight on position information to match its validity as a cue to word meaning. The tendency toward selective attention to position information in the extreme frequency conditions may also reflect optimization: Apparently, position had become reliable enough as a cue to word meaning to induce exclusive attention on a few trials without increasing the overall error rate substantially.

Note that what was optimized was the minimum RTs in a block of trials; otherwise, the facilitation with frequent stimuli was balanced by the interference with infrequent stimuli. Except for the 20\% condition, overall RTs and error rates (i.e., means from compatible and conflicting trials weighted by their frequencies) stayed relatively constant. Performance was better overall in the $20 \%$ condition than in the other frequency conditions. RTs and error rates (in parentheses) were $479(.06), 507(.04), 495(.06)$, and $500(.06)$ for the $20 \%, 40 \%, 60 \%$, and $80 \%$ conditions, respectively. Nevertheless, the results provide evidence that subjects followed the "metastrategy" of assigning weights in proportion to cue validity. Experiment 3 provides further evidence. More extreme relative frequencies (10\% and $90 \%$ ) were included in an attempt to induce subjects to attend exclusively to the unreported dimension.

\section{EXPERIMENT 3}

\section{Method}

Subjects. Eight undergraduates from Erindale College participated to fulfill course requirements. Six were female and two were male. None had served in the previous experiments.

Procedure. The apparatus and stimuli were the same as in Experiments 1 and 2. The procedure was the same as in Experiment 2, except that the four relative frequencies were $10 \%, 20 \%, 80 \%$, and $90 \%$.

\section{Results}

Mean RTs for correct responses in each combination of trial type and frequency are plotted in Figure 3.

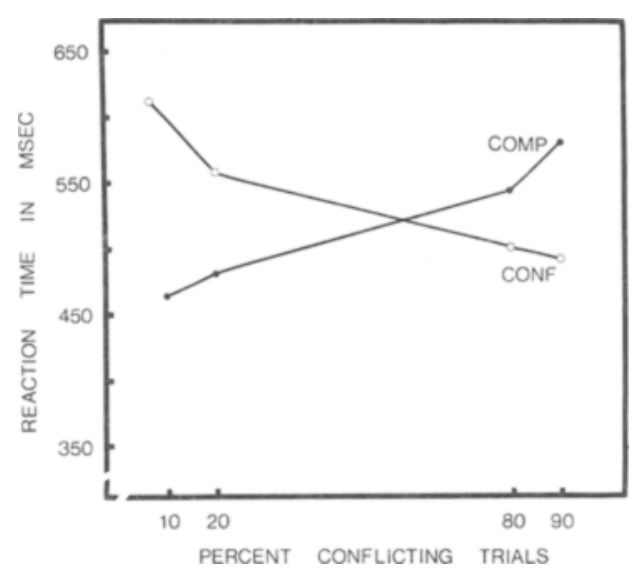

Figure 3. Reaction time as a function of the relative frequency of conflicting trials (percentage) in Experiment 3. (Trial type is the parameter: Filled circles $=$ trials with compatible stimuli; open circles $=$ trials with conflicting stimuli.)

For the third time, there was an effect of trial type modulated by frequency. RTs to compatible stimuli increased monotonically with the relative frequency of conflicting trials, while RTs to conflicting stimuli decreased. The crossover interaction replicated once again, and it remained asymmetrical, even in the extreme frequency conditions. The difference between conflicting and compatible trials in the $90 \%$ condition $(89 \mathrm{msec})$ was smaller than the difference in the $10 \%$ condition $(159 \mathrm{msec})$, and the difference in the $80 \%$ condition ( $43 \mathrm{msec}$ ) was smaller than the one in the $20 \%$ condition $(75 \mathrm{msec})$. Note that the differences in the $20 \%$ and $80 \%$ conditions agree well with values obtained in Experiments 1 and 2.

Apart from some difficulty discriminating highfrequency conditions from each other and lowfrequency conditions from each other, the major trends were reflected in individual subject data. Seven out of eight subjects showed a monotonic decrease in the difference between conflicting and compatible RTs as the relative frequency of conflicting trials increased. The one anomalous subject showed a larger difference in the $20 \%$ condition than in the $10 \%$ condition. Eight out of eight subjects showed faster RTs to conflicting stimuli than compatible stimuli in the $90 \%$ condition, and six out of eight showed it in the $80 \%$ condition.

Analysis of variance showed that frequency had no significant main effect $[F(3,21)<1]$ and the effect of trial type was weak, although significant $[F(1,7)=5.68$, $\mathrm{p}<.05$ ], but the interaction between them was highly significant $[F(3,21)=71.76, p<.01]$. The facilitation and interference observed in the extreme frequency conditions (10\% and $90 \%)$ were compared to those observed in the intermediate frequency conditions (20\% and $80 \%$ ) to test the hypothesis that weights are adjusted in proportion to cue validity to optimize performance. The relevant contrast was significant $[F(1,21)=12.32, p<.01]$, indicating more facilitation 
and interference in extreme frequency conditions than in intermediate frequency conditions.

The error rates, presented in Table 1, reflect similar trends. Note that there was a tendency toward higher error rates with infrequent stimuli in the extreme frequency conditions.

\section{Discussion}

This experiment replicated once again the crossover interaction observed in Experiments 1 and 2, suggesting that attention is divided between dimensions even with extreme relative frequencies ( $10 \%$ and $90 \%$ conditions). There was some evidence for selective attention to the unreported dimension in the $10 \%$ and $90 \%$ conditions, but it occurred on no more than $8 \%$ and $15 \%$ of the trials, respectively. (Again, these estimates represent the difference in error rate between frequent and infrequent trial types.) It is interesting that the tendency toward selective attention to the unreported dimension was substantially smaller in the $20 \%$ and $80 \%$ conditions, where a stronger effect had been observed in Experiment 2 . This suggests a range effect whereby subjects use the strategy only in the most extreme frequency conditions they experience.

The greater facilitation and interference in the $10 \%$ and $90 \%$ conditions support the hypothesis that subjects adjusted the weight on position information to match its validity as a cue to word meaning. Again, it was minimum RT that seemed to be optimized; facilitation and interference seemed to balance each other. Again, overall RTs and error rates (means weighted by their frequencies) were relatively constant across conditions. RTs and error rates (in parentheses) were $479(.020)$, $503(.019), 519(.024)$, and $502(.026)$ for the $10 \%$, $20 \%, 80 \%$, and $90 \%$ conditions, respectively. Like those of Experiment 2, these results suggest that subjects followed the "metastrategy" of assigning weights in proportion to cue validity. The point that the three strategies involving selective and divided attention may be products of one such "metastrategy" appears well taken.

\section{GENERAL DISCUSSION}

The present experiments have shown that performance in Stroop-like tasks may depend on strategy and perhaps may be more flexible than had been thought previously. Nevertheless, it was clear in each experiment that relatively inflexible strategy-invariant components were also important. The crossover interaction that indicated strategic flexibility was asymmetrical in all three experiments. With cue validity held constant, facilitation and interference were greater with compatible stimuli than with conflicting stimuli. As noted before, this may indicate that subjects are reluctant to assign large negative weights. However, if subjects are assumed to assign weights in the same proportion to cue validity regardless of sign, the asymmetries can be exploited to reveal the relative strength of weights assigned automatically and attentionally to the unreported dimension. When conflicting stimuli are rare (e.g., 10\%, 20\%, and 40\% conditions), attentional weights and automatic weights have the same sign, so that RT differences between infrequent conflicting trials and frequent compatible trials will reflect the effects of two positive attentional weights and two positive automatic weights. When conflicting stimuli are frequent (e.g., 60\%, 80\%, and 90\% conditions), however, attentional weights and automatic weights will have opposite signs, and RT differences between infrequent compatible trials and frequent conflicting trials will reflect the effects of two positive attentional weights and two negative automatic weights. Thus, for conditions of equal cue validity (i.e., $10 \%$ and $90 \%, 20 \%$ and $80 \%$, and $40 \%$ and $60 \%$ ), the effects of attentional weights can be estimated as $25 \%$ of the sum of these differences [i.e., $\left(\mathrm{RT}_{\mathrm{I}, \mathrm{Cf}}-\mathrm{RT}_{\mathrm{F}, \mathrm{Cp}}\right)+$ $\left(R_{\mathrm{I}, \mathrm{Cp}}-\mathrm{RT}_{\mathrm{F}, \mathrm{Cf}}\right)$, where $\mathrm{F}=$ frequent, $\mathrm{I}=$ infrequent, $\mathrm{Cf}=$ conflicting, and $\mathrm{Cp}=$ compatible $]$, and the effects of automatic weights can be estimated as $25 \%$ of the difference between them [i.e., $\left(\mathrm{RT}_{\mathrm{I}, \mathrm{Cf}}-\mathrm{RT}_{\mathrm{F}, \mathrm{Cp}}\right)-$ $\left.\left(R T_{I, C p}-R_{F, C f}\right)\right]$. The effects, estimated in this manner for each experiment, are presented in Table 2.

In conditions where it was apparent that subjects overcame habitual response tendencies, the effects of attentional weights were larger than those of automatic weights. Note that these constraints on the weights follow from logical consideration of the random-walk model presented in the introduction. Further, the effects of attentional weights increased with cue validity, while the effects of automatic weights remained relatively constant. Together, these trends suggest that attentional weights are adjusted strategically in proportion to cue validity and that automatic weights are beyond the subject's control.

A general point emerging from those studies is that attention is paid to information to the extent that it is available early enough to be considered (i.e., position information interfered with word information but not vice versa) and to the extent that it predicts responses (external or internal) appropriate to the trial. The same point has been made in the literature on priming, where cue validity and the time at which the prime is presented

Table 2

Effects (in Milliseconds) of Weights Assigned Atten tionally and Weights Assigned Automatically in Experiments 1, 2, and 3

\begin{tabular}{ccccccc}
\hline & \multicolumn{5}{c}{ Frequency (Percent) } \\
\cline { 2 - 7 } & \multicolumn{5}{c}{ Experiment } \\
\cline { 2 - 7 } Effect & $10-90$ & $20-80$ & $\frac{20-80}{2}$ & $20-80$ & $40-60$ \\
\hline Automatic & 18 & 8 & 10 & 8 & 14 \\
Attentional & 62 & 30 & 31 & 37 & 17 \\
\hline
\end{tabular}


relative to the primed stimulus have important effects on performance (e.g., Neely, 1977; Posner \& Snyder, 1975b). Indeed, the advantage for conflicting stimuli observed in the $80 \%$ and $90 \%$ conditions of the present experiments resembles Neely's (1977) finding that subjects could benefit by being primed with a category that differed from the category of the target word but predicted it reliably (i.e., when primed with BODY, the target was likely to be a part of a building). Moreover, the present disadvantage for compatible stimuli in the $80 \%$ and $90 \%$ conditions resembles Neely's finding of interference when the prime led subjects to expect a word from a different category but the target was a word from the same category (i.e., when primed with BODY, a part of a building was expected, but a part of a body was presented). Perhaps the literature on priming effects and the voluminous literature on Stroop-like effects are more closely related than had been thought previously (cf. Posner \& Snyder, 1975a). Both are consistent with the view that performance depends on a strategic compromise between instructions, the task environment, and the (automatic) cognitive resources the subject brings to the task (see Logan, 1978).

An important question for future research is the extent to which the strategy of dividing attention between reported and unreported dimensions pervades in other Stroop-like tasks. Possibly, a significant portion of the literature may have been interpreted incorrectly. We know that divided attention extends beyond the current procedure (which involves repeating a few stimuli many times) to situations in which stimuli are never repeated. We have unpublished data showing a crossover interaction in a task in which subjects verify addition equations (e.g., $3+4=7$ ). Each of the 80 possible combinations of the digits 1 to 9 (excluding $2+2$ ) was presented once as a positive stimulus and once as a negative stimulus. The negative stimuli were either confusable (e.g., $3+4=12$, which would be true for multiplication) or not confusable (e.g., $3+4=11$, which is never true). When $20 \%$ of the negative stimuli were confusable, the typical Stroop effect occurred: Because $3+4=12$ seemed true, it took longer to reject than $3+4=11$. But when $80 \%$ of the negative stimuli were confusable, confusable stimuli were rejected faster than nonconfusable stimuli, reversing the Stroop effect. We are also investigating cuing effects independent of stimulus frequency (using a priming stimulus), and cue validity effects in tasks with more irrelevant dimensions.

A final comment on the random walk is in order. Predictions drawn from logical consideration of qualitative features of the model have done an admirable job of accounting for performance in the present experiments. A more detailed quantitative fit does not seem necessary in the present context because the n:mber of parameters to be estimated (two drift rates, two thresholds, and three weights) approaches the number of data points. A more stringent test of the random-walk model might involve examining speedaccuracy tradeoff functions (see Pachella, 1974) in Stroop-like tasks. The hypothesized shift in the initial state of evidence about word meaning that occurs when information about position becomes available might be observed in the early portion of the function. Nevertheless, the present application of the random walk has been encouraging in that three strategies, each with distinct empirical consequences, have been identified as variations of the same process. This implicates such processes as basic elements in the mechanics of thought, modifiable by strategy to afford optimal performance.

\section{REFERENCES}

Dixon. P. Numerical comparison processes. Memory \& Cognition, 1978, 6. 454.461.

DYER. F. N. The Stroop phenomenon and its use in the study of perceptual, cognitive, and response processes. Memory \& Cognition. 1973, 1. 106-120.

Kahneman, D. Attention and effort. Englewood Cliffs, N.J: Prentice-Hall, 1973

KEELE, S. W. Attention demands of memory retrieval. Journal of Experimental Psychology. 1972. 93, 245-248.

Logan, G. D. Attention in character classitication: Evidence for the automaticity of component stages. Joumal of Experimental Psychology: General, 1978, 107, 32-63.

NfELy, J. H. Semantic priming and retrieval from lexical memory: Roles of inhibitionless spreading activation and limited-capacity attention. Journal of Experimental Psychology: General, 1977. 106. 226-254

PACHELla. R. G. The interpretation of reaction time in information-processing research. In B. H. Kantowitz (Ed.), Human information processing: Tutorials in performance and cognition. Hillsdale. N.J: Erlbaum, 1974.

PAlfF. S. R. Judging pictorial and linguistic aspects of space. Me'mon' \& Cognition, 1978, 6. 70-75.

PalkF. S. R.. \& Orson. D. R. Spatial and verbal rivalry in a Stroop-like lask. Canadian Journal of Psychology, 1975, 29. 201-204.

Posner, M. I. d S S Syder, C. R. R. Attention and cognitive control. In R. L. Solso (Ed.). Intormation processing and cognition: Th. Loyola symposium. Potomac, Md: Erlbaum, 1975. (a)

Posner. M. I.. \& SNYder. C. R. R. Facilitation and inhibition in the processing of signals. In P. M. A. Rabbitt \& S. Dornic (F.ds.). Anemion and performance $V$. New York: Academic Press, 1975. (b)

STROOP. J. R. Studies of interference in serial verbal reactions. Journal of Experimental Psychology, 1935, 18, 643-662.

TrFisman. A. M. Strategies and models of selective attention. Psychological Re'vi'w. 1969. 76. 282-299.

\section{NOTES}

1. In general, attention to the unreported dimension will affect the drift rate in the random walk. In the present case, simplification seems warranted because the bulk of the effect of the unreported dimension will have finished before the major effect of the reported dimension begins. Note that we do not intend to localize interference at any stage in the standard stage analysis of the task; encoding, comparison, and decision stages are aggregated in the random-walk model.

2. A tempting speculation is that weights increase in 
magnitude in proportion to the amount of attentional capacity invested in processing the reported dimension (i.e., attention controls perceptual processing directly). This would introduce constraints on the weights attached to the reported and unreported dimensions (i.e., their sum can be no greater than some constant representing the limit on capacity), which may prove useful in interpreting the data. However, we believe that weights are parameters that control the contribution of perceptual systems (i.e., attention controls perceptual processing indirectly, mediated by parameters; see Logan, 1978), and as such, each weight requires about the same amount of attentional capacity to be maintained, regardless of its magnitude.

(Revision accepted for publication February 26, 1979.) 